The Official Journal of the Kettil Bruun Society for Social and Epidemiological Research on Alcohol

\title{
Alcohol's harm to others: An international collaborative project
}

\author{
Sarah Callinan ${ }^{1}$, Anne-Marie Laslett ${ }^{1,2,3,4}$, Dag Rekve ${ }^{5}$, Robin Room ${ }^{1,6}$, Orratai Waleewong ${ }^{4,7}$, Vivek Benegal ${ }^{8}$, Sally
} Casswell $^{9}$, Ramon Florenzano ${ }^{10}$, Hoang Thi My Hanh ${ }^{11}$, Vu Thi Minh Hanh ${ }^{12}$, Siri Hettige ${ }^{12,1^{3}}$, Taisia Huckle ${ }^{9}$, Akanidomo Ibanga $^{14}$, Isidore Obot ${ }^{15,16}$, Girish Rao ${ }^{17}$, Latsamy Siengsounthone ${ }^{18}$, Georgia Rankin ${ }^{1,3}$, and Thaksaphon Thamarangsi ${ }^{19}$

${ }^{1}$ Centre for Alcohol Policy Research, School of Psychology and Public Health, La Trobe University, Bundoora, Australia

${ }^{2}$ National Drug Research Institute, Curtin University, Perth, Australia

${ }^{3}$ Eastern Health Clinical School, Monash University, Clayton Australia

${ }^{4}$ School of Population and Global Health, University of Melbourne, Victoria, Australia

${ }^{5}$ Mental Health and Substance Abuse, WHO, Geneva, Switzerland

${ }^{6}$ Centre for Social Research on Alcohol and Drugs, Stockholm University, Sweden

${ }^{7}$ International Health Policy Program, Ministry of Public Health, Nonthaburi, Thailand

${ }^{8}$ Centre for Addiction Medicine, National Institute of Mental Health and NeuroSciences, Bangalore, India

${ }^{9}$ SHORE and Whariki Research Centre, School of Public Health, Massey University, Auckland, New Zealand

${ }^{10}$ Universidad del Desarrollo, Servicio de Psiquiatria Hospital del Salvador, Santiago de Chile, Chile

${ }^{11}$ Health Strategy and Policy Institute, Ministry of Health, Vietnam

${ }^{12}$ Department of Sociology, University of Colombo, Colombo, Sri Lanka

${ }^{13}$ Globalism Research Centre, School of Social Sciences, RMIT University, Melbourne, Australia

${ }^{14}$ School of Psychology, University of Birmingham, Birmingham, UK

${ }^{15}$ Department of Psychology, University of Uyo, Uyo, Nigeria

${ }^{16}$ Centre for Research and Information on Substance Abuse (CRISA), Uyo, Nigeria

${ }^{17}$ Centre for Public Health, National Institute of Mental Health and NeuroSciences, Bangalore, India

${ }^{18}$ Research Outcomes Management Department, National Institute of Public Health, Ministry of Health, Vientiane Capital, Lao PDR

${ }^{19}$ Non-Communicable Diseases and Environmental Health, WHO Regional Office for South-East Asia Region, New Delhi, India

\section{Abstract}

Aims: This paper outlines the methods of a collaborative population survey project measuring the range and magnitude of alcohol's harm to others internationally.

Setting: Seven countries participating in the World Health Organization (WHO) and ThaiHealth Promotion Foundation (ThaiHealth) research project titled "The Harm to Others from Drinking," along with two other countries with similar studies, will form the core of a database which will incorporate data from other countries in the future.

Measures: The WHO-ThaiHealth research project developed two comparable versions of a survey instrument, both measuring harm from others' drinking to the respondent and the respondent's children.

Design: Surveys were administered via face-to-face methods in seven countries, while similar surveys were administered via computer-assisted telephone interviews in two additional countries. Responses from all surveys will be compiled in an international database for the purpose of international comparisons.

Discussion: Harms from the alcohol consumption of others are intertwined with the cultural norms where consumption occurs. The development of this database will make it possible to look beyond reports and analyses at national levels, and illuminate the relationships between consumption, harms, and culture.

Conclusions: This database will facilitate work describing the prevalence, patterning, and predictors of personal reports of harm from others' drinking cross-nationally. 
Traditionally, the focus of attention in studying problems from drinking has been on drinkers' own problems - their harms, addictions, and treatment (Babor et al., 2010; Levine, 1985; Room et al., 2010); and a small number of specific harms to others (HTO) from drinking - namely drink driving, physical assault, and fetal alcohol spectrum disorders (Alati et al., 2006; Connor \& Casswell, 2009; Graham et al., 2008). While some elements of HTO from drinking have been captured in population surveys since the mid-1980s, a broader approach that spanned the range of harms experienced by people from the drinking of others was required.

The first large scale studies with this broader view on HTO were conducted in Australia in 2008 and New Zealand in 2009 (Casswell, Harding, You, \& Huckle, 2011; Laslett et al., 2010). Also in 2009, was a meeting organised by the World Health Organization (WHO) in Stockholm to initiate "an international collaborative research initiative on Alcohol, Health and Development." During that meeting, HTO was designated as a priority program area and included in WHO's "Global Strategy to Reduce the Harmful Use of Alcohol" (World Health Organization, 2010). These efforts received substantial attention, not just in the media but also in research and health promotion institutes around the world, and led to similar survey data being sought internationally.

\section{WHO-ThaiHealth Project}

In 2011, the WHO and ThaiHealth Promotion Foundation (ThaiHealth), an independent state agency (created by the Health Promotion Act 2001 and funded by 2\% surcharge tax of tobacco and alcohol excise taxes), funded a multi-site project to pioneer the measurement and analysis of HTO from drinking in low- and middle-income countries. This project included data collection in national studies in Thailand, Chile, India, Nigeria, Sri Lanka, and Vietnam. Furthermore, a team in the Lao People's Democratic Republic (LPDR) has been supported by ThaiHealth to implement the WHO-ThaiHealth project protocol.

This paper outlines the methods of the international collaborative research project, led by the WHO and ThaiHealth, involving not only the seven countries mentioned above, but also including existing survey data from Australia and New Zealand. The cross-national comparative project will be used to highlight the burden of harm experienced by those other than the drinker in a wide range of cultures and contexts, and to analyse the correlates and conditions of the occurrence of harm to suggest means of prevention and response.

The main six-country study is divided into two phases; the first phase includes a scoping and assessment study and a general population survey of at least 1,500 completed interviews of adults. Each national team writes and publishes a descriptive report for the country on the national results. The archive will be used to facilitate cross-national analysis of the results across societies. A master protocol has been developed for the first phase of the WHO-ThaiHealth project, drawing on the approaches and experiences of the Australian and New Zealand studies.
Each collaborating country that carries out the project agrees to follow one of two options in the master research protocol. The second phase of the WHO project will, if feasible, include register data analyses of HTO from drinking, and agency caseload studies in three firstresponse agency systems (e.g., police, child welfare agencies, and emergency health services).

\section{Nine-Country Archive of HTO Survey Data}

After securing funding from the National Health and Medical Research Council (NHMRC) in Australia, work has begun on building an archive of survey data from the seven countries listed above, and Australia and New Zealand. This paper focuses on the survey component in the first phase of the WHO-ThaiHealth project, and on the equivalent Australian and New Zealand surveys. The collection of data from different countries in a largely comparable form offers the potential of using the data not only for analyses in a national frame to guide local policy and action, but also for cross-societal comparative analysis. The population survey data of the first phase of the WHOThaiHealth project is, by its nature, better suited for comparison between countries than data describing the institutions responding to community problems and their clients, which is less likely to be comparable between countries.

Conduct of standardised research enables the establishment of a comparable evidence base for policies aiming to reduce rates of harm, and affords the opportunity for crossnational examination of the interplay between drinking cultures, consumption, and HTO. The effectiveness of such a strategy has been demonstrated by another international project titled "Gender, Alcohol and Culture: An International Study" (GENACIS). This study used comparable surveys from 36 countries to illuminate the relationships between gender, alcohol, and culture with much success (Rahav, Wilsnack, Bloomfield, Gmel, \& Kuntsche, 2006; Wilsnack, Wilsnack, Kristjanson, Vogeltanz-Holm, \& Gmel, 2009).

The WHO-ThaiHealth project research team (including the Australian investigators), was tasked with designing a survey that could be used internationally to measure HTO. In the end two versions of the survey, based on the Australian and New Zealand instruments, were developed. This paper describes the WHO-ThaiHealth versions of the questionnaire, with some reference to the earlier Australia/New Zealand instruments. A data archive, funded by the NHMRC and the WHO, containing data from the nine participating countries and available for collaborative use is currently being compiled. This international database will be developed and maintained in Australia, but available for analytical use by any group of participating teams. Data from other countries with similar survey methodologies will be added to the archive as they become available. 


\section{Design}

\section{Conceptual approach}

The conceptual basis of current HTO surveys is described in Room et al. (2010). It draws on understandings of alcohol's potential impact on major social roles, including those as family or household members (e.g., parents, carers for children), relatives, friends, and co-workers, and alcohol's potential impact on interactions with others not known to the respondent, which in this paper is termed "strangers" (Klingemann \& Gmel, 2001).

Traditionally, alcohol surveys have primarily asked drinkers themselves about problems from their own drinking (Room, 2000). These have included questions which imply HTO, but the responses have usually been analysed from the perspective of the problems for the drinker. When, for instance, the drinking results in the drinker losing a job or being divorced, this has conventionally been analysed without consideration of the other parties harmed. While some such items in existing surveys can be reanalysed from the perspective of HTO, the items are often non-specific and the topic coverage is limited. Also, people under-report harms their drinking may have inflicted on others compared to experienced harms due to someone else's drinking (Callinan \& Room, 2014). Furthermore, there are a range of harms that others may experience that the person responsible may not recognise, for instance those surrounding fear, litter, and noise.

Harms from the drinking of others can be broadly split into three groups based on the relationship between the harmed party and the drinker: harms from known drinkers, harms to children, and harms from strangers.

Harms from known drinkers. Harms from drinkers known to the respondent tend to be organised in terms of the relationship of the drinker with the respondent: partners, family members in the household, family members outside of the household, friends, work colleagues, acquaintances, and neighbours. In particular, the difference between harms from a drinker who lives or does not live with the respondent is an important distinction in the study.

Harm to children. Due to ethical considerations, most countries did not have participants below the age of 18 , with none under the age of 12 . Therefore, harms attributable to adults' drinking experienced by children were assessed by asking the respondent about children (aged 17 or below) in the care of the respondent. Respondents in the WHO-ThaiHealth project were asked about harm to children from their own drinking, as well as others' drinking.

Harm from strangers. Respondents were also asked about a range of specific and subjective experiences of harm from strangers or people not well known to the respondent. This includes what is termed in Australia "amenity harms", that is, harms where the drinker did not specifically interact with the harmed party (e.g., leaving empty bottles and litter, making noise late at night) and also less tangible harm such as fear (e.g., where someone feels they need to avoid an area because of the presence of drinkers) (Callinan \& Room, 2014). More tangible harms such as damage to property, physical assault, and confrontations that put the respondent in fear are also assessed.

\section{Surveys}

The WHO-ThaiHealth project version 1 and 2 survey templates were developed together and are broadly comparable; however, they also meet two different aims. Version 1 enables more detailed prevalence and economic estimates of harm to others. For example, in addition to questions on whether harms occurred, Version 1 includes indications of how frequently they occurred, and if material damage was incurred, how much the item was worth. Version 2 has more detailed items on the respondent's own drinking and drinking networks to put the harm in context, and enables comparisons with survey data from GENACIS (Wilsnack et al., 2009). The Australian and New Zealand surveys preceded the WHO-ThaiHealth project and were closer to Version 2, but differ in some respects from the later surveys, which were able to draw on the experience of the earlier studies. The information below highlights the key differences to be aware of when comparing analyses between these surveys:

Harms to respondent. In the case of harms to the respondent from known drinkers, co-workers, strangers, and to the respondent's children, subjective measures of harm were included in most surveys. In Version 1 and 2, respondents were asked to measure the impact of this harm on a scale of $1-10$ (where 1 is a little and 10 is a lot). While the Australian survey does not ask the overall impact on a scale of 1-10, it does ask if respondents were impacted a little or a lot, which can be roughly converted into a 1-10 score (Callinan, 2014). New Zealand does not contain a subjective measure of harm.

Demographics. Both survey versions ask about demographic and household information. With different countries undertaking these surveys there are obvious questions that will require country-specific responses. Ethnicity, ancestry, region of residence, household income, educational level, occupation, and religious preference will yield different responses depending on the country of survey. Such variables not only allow description and comparison of the social location of patterns and problems in national populations, but can also be used in multivariate analyses accounting for country-level differences in harms and examining variations between countries in the demography of harms from others' drinking. Version 2 asks additional questions to women about alcohol and pregnancy.

Drinking variables. Questions on the respondent's own drinking consumption and associated impacts are included in both survey versions; however, Version 2 is more extensive. Both versions include quantity/frequency questions, some items on heavy episodic drinking, and whether the respondent's drinking has negatively affected anyone else, with the extent of effect measured on a scale 
of 1-10. The Australian survey contains comparable questions, except that the effect of the respondent's drinking on others is asked in summary terms. The New Zealand questions on consumption are qualitatively different from those in the other surveys; however, percentile-based groupings of heavier drinkers can still be used for some analyses. That is, respondents would be ranked based on total volume and then placed in groups based on high, medium, or low consumption within each country for comparisons.

Version 2 asks additional questions regarding drinking contexts and outcomes of the respondent's own drinking (e.g., drinking consequences to self and family from their own perspective) (Graham et al., 2011), and asks all items from the Alcohol Use Disorders Identification Test (AUDIT) (Saunders, Aasland, Babor, De la Fuente, \& Grant, 1993). Furthermore, there are questions about the settings within which people drink, in addition to attitudes towards alcohol and intoxication in varying social settings. Many of these items are comparable with items in the GENACIS surveys (Wilsnack et al., 2009).

Questions asking respondents about their health, healthseeking behaviour, and drug use are included in Version 2, but not in Version 1. However, both surveys ask for respondents' satisfaction with their life as a whole on a scale of 1-10. Version 1 asks the eight items on the Personal Wellbeing Index (International Wellbeing Group, 2013). Both surveys complete the EUROQoL-5D (The EuroQol Group, 2009). The Australian and New Zealand survey questions on health and wellbeing are equivalent to Version 1.

Brief assessment of harms. The majority of this section is made up of questions about specific experiences of harm at the hands of strangers or known drinkers (this differentiation is made). This section was developed to bridge the gap between the more detailed surveys of this study, and pre-existing short-list survey questions in Europe (Huhtanen \& Tigerstedt, 2012) and North America (Eliany, Giesbrecht, Nelson, Wellman, \& Wortley, 1992; Greenfield et al., 2009). A brief assessment of alcoholrelated harms experienced from other people's drinking, primarily drawn from this section, is present in both versions of the WHO-ThaiHealth project surveys. But, in this section and in later ones, when asked whether a particular event has occurred, Version 2 respondents are asked to answer yes or no; whereas Version 1 responses are categorised into "no," "yes, 1-2 times," or "yes, 3 or more times.”

Both surveys contain the 13 core questions, but Version 1 also includes several follow-up questions relating to expenses incurred from the events which involve property damage or other tangible costs. Respectively, the Australian and New Zealand surveys have 10 and seven comparable questions to the 13 core questions mentioned above. Some of these items about harm from known drinkers were specific to the most harmful drinker in the respondent's life, not any harmful drinker. Therefore, as will be made clear in any analysis that the prevalence of these harms from Australia and New Zealand will be an underestimate compared to other countries. The brief assessment of harms is particularly important for international comparisons, as assessments of suitability for any new countries hoping to become part of the data archive will be based on this brief assessment. This brief assessment will thus become the anchor for international comparisons of the prevalence of alcohol's HTO.

Heavy drinkers. A defining component of both versions of the WHO-ThaiHealth surveys is the section asking about people in the respondent's life whom the respondent would consider to be a fairly heavy drinker, or someone who drinks a lot sometimes. Respondents are asked the relationship of these people to the respondent, whether they live in the respondent's household, and if their drinking has negatively affected the respondent in the last 12 months. Respondents who reported being harmed by the drinking of one or more such persons were then asked 10 items on specific harms which occurred in the last 12 months. These can be analysed with the 11 items on harms from family members or friends in the brief assessment section (see above). Version 1 includes an additional follow-up question quantifying the amount of money associated with friends or family taking money or damaging valuables belonging to the respondent due to their drinking. Those respondents who identified a heavy drinker in their life were further asked to identify the relationship of the person whose drinking most negatively affected them in the past year, along with information about the identified drinker's sex, age, and alcohol consumption. The Australian survey asked these questions differently from the WHOThaiHealth versions, with more prompting of relationship types and higher caps in the possible number of heavy drinkers reported, which may result in higher reported numbers of heavy drinkers in this country. Caution will be required in cross-national analysis involving the Australian data on these variables.

Caring for drinkers. A series of questions in both WHOThaiHealth surveys asks respondents about things they may have done to care for the drinkers in their life. Version 2 simply asks whether the events occurred. Version 1 is more detailed, allowing an approximation of the time spent caring in hours, days, or weeks. The New Zealand survey asks three of the four caring questions, but does not include indicators for cost or time spent caring. Both Australia and New Zealand use different, but comparable, response categories to Version 1.

Harms to children. As described above, harms to children from others' drinking are also assessed. Those respondents identified as having responsibility for children under 18 years of age were asked a series of questions about events the child/children may have experienced due to another's alcohol use. Both versions of the WHO-ThaiHealth surveys contain a subjective measure of the degree to which the child or children were negatively affected by someone's drinking. Australia and New Zealand have comparable questions for majority of the child harm questions, but they use different response categories. Version 1 and 2 also ask whether the respondent's own 
drinking or others' drinking affected these children, whereas the Australian and New Zealand surveys did not.

Work harms. Those respondents who were in the labour force were asked about work-related problems due to colleagues' drinking. Both WHO-ThaiHealth versions ask the same questions and include a subjective measure of the negative impact caused. Version 1 subsequently asks those respondents who report having worked extra hours the amount of extra time taken in hours or days. Australia and New Zealand have several comparable questions. Australia also includes a subjective measure asking whether respondents were impacted a little or a lot by their colleagues' drinking.

Community harms. The impacts and potential harm caused from strangers' drinking are also ascertained from several questions in the WHO-ThaiHealth surveys, ranging from public nuisance to physical abuse. These items can be analysed along with seven items from the brief assessment section (see above). Besides the questions on specific events, the respondent was asked the extent of the overall negative impact on him or her, from the drinking of people not known to him or her. Of the four core questions asking about stranger harms, Australia and New Zealand each have three comparable questions.

Service use. In the WHO-ThaiHealth surveys, respondents are asked about the services they may have sought for help or utilised because of harms experienced from someone else's drinking (e.g., police, hospitals, other medical services, counselling). Version 2 asks whether each service was used, and a summary question on the number of days taken off work, school, or out of the respondent's normal routine because of others' drinking. Version 1 is more detailed, allowing an approximation of the time and money lost. The Australian and New Zealand surveys ask each of the core service use questions, except that New Zealand does not have comparable data about time or cost relating to respondents' service use. Improving on the Australian and New Zealand versions, an additional question was added to Versions 1 and 2 about use of self-help groups and informal assistance.

\section{Ethics}

The WHO-ThaiHealth project's master protocol, including the two survey instrument versions, was approved by the WHO Ethics Review Committee, and ethics approval was also sought and received from the appropriate Human Research Ethics Board in each participating country. This clearance was not just for the data collection and dissemination, but also for the data to be stored at the Centre for Alcohol Policy Research in Australia, where ethics approval for the international project was also granted.

\section{Data Collection}

The dates of data collection, sample size, regions, age range, and questionnaire and response rate in all participating countries in this project are shown in Table 1. Data collection has been completed in all countries. As can be seen, the response rates on the whole are quite high, except in Australia. This response rate was in the normal range for telephone surveys conducted in Australia, which have relatively low response rates (O'Toole, Sinclair, \& Leder, 2008). Four countries used Version 1 of the survey and three used Version 2. Australian and New Zealand surveys were administered before the development of these surveys and are generally comparable with Version 1.

Face-to-face interviews were conducted in seven of the countries, where interviewers used iPads to administer the surveys and collect the data except for Vietnam, Thailand and India where pen and paper was used to write down the answers. At each site, interviewers completed rigorous training to ensure that administration of the surveys was uniform and ethically appropriate. In the two countries where the survey was administered by phone, computerassisted telephone interviewing is the norm and both sites used well established research centres that specialised in this type of work. Pre-weights, based on the likelihood of being interviewed on the basis of household composition, will be available in all countries. Where available and appropriate, post-weights will also be applied to match the appropriate census distributions on the basis of age, sex, and geographical location. As response rates were high in many of these countries, the need to post-weight is reduced.

Table 1

Data collection methods from each of the nine countries contributing to the data archive

\begin{tabular}{|c|c|c|c|c|}
\hline \multirow[b]{2}{*}{ Country } & \multirow[b]{2}{*}{ Data Collection } & \multicolumn{2}{|c|}{ Respondents } & \multirow[b]{2}{*}{ Sampling scope } \\
\hline & & Planned & Actual & \\
\hline Chile & Oct 12-Sep 13 & 1500 & 2238 & 7 cities \& surrounding areas \\
\hline Nigeria & Oct 12-Dec 13 & 2000 & 2270 & 3 states ( 1 in North, and 2 in South) \\
\hline India & Dec 13-Aug 14 & 2800 & 3302 & 4 regions in Karnataka State \\
\hline Sri Lanka & Sep 13-Feb 14 & 1650 & 2475 & National (9 provinces, 21 districts) \\
\hline Thailand & Sep 12-Mar 13 & 1800 & 1695 & National (4 regions \& Bangkok, 5 provinces, 15 districts) \\
\hline Vietnam & Dec 12-May 13 & 1512 & 1501 & 6 regions, 1 province per region \\
\hline Laos & Oct 13 -Nov 13 & 1260 & 1257 & National (3 regions, 3 provinces, 6 districts) \\
\hline Australia & Oct 08-Dec 08 & 2500 & 2649 & National (7 states and 2 territories) \\
\hline New Zealand & Sep 08-Mar 09 & 3000 & 3068 & National \\
\hline
\end{tabular}




\begin{tabular}{lllcccc}
\hline Country & Age & \multicolumn{1}{c}{ Questionnaire } & Administration & Response rate & Pre-weights & Post-weights \\
\hline Chile & $18+$ & Version 1 & F2F & $72 \%$ & HH & None \\
Nigeria & $18-64$ & Version 2 & F2F & $99 \%^{\mathrm{a}}$ & $\mathrm{HH}$ & None \\
India & $18+$ & Version 1 & F2F & $97 \%$ & $\mathrm{HH}$ & None \\
Sri Lanka & $18-64$ & Version 2 & F2F & $93 \%$ & $\mathrm{HH}$ & None \\
Thailand & $18-70$ & Version 1 & F2F & $94 \%$ & $\mathrm{HH}$ & None \\
Vietnam & $18+$ & Version 1 & F2F & $99 \%$ & $\mathrm{HH}$ & None \\
Laos & $15-64$ & Version 1 & F2F & $99 \%$ & $\mathrm{HH}$ & None \\
Australia & $18+$ & Australian version & CATI & $35 \%$ & $\mathrm{HH}$ & $\mathrm{A} / \mathrm{S} / \mathrm{G}$ \\
New Zealand & $12-80$ & New Zealand version & CATI & $64 \%$ & $\mathrm{HH}$ & $\mathrm{A} / \mathrm{S} / \mathrm{G} / \mathrm{E}$ \\
\hline
\end{tabular}

Note. F2F = Face to face; CATI = Computer Assisted Telephone Interviewing; HH = Household; A/S/G/E = Age, sex, geographical location, ethnicity

${ }^{\mathrm{a}} \mathrm{A}$ response rate of $99 \%$ was reported among households where someone was home.

\section{Limitations of the Survey}

In some countries, it was decided on pragmatic grounds to sample within a state or region of the country, rather than across the country. This needs to be taken into account when interpreting results and will be noted in all published work. Furthermore, response rates are those reported by fieldwork directors and may not have consistently applied rules on randomisation in the household.

There are cultural differences which cannot be entirely controlled for, such as in nuances of language and in varying thresholds of noting behaviour and in its ascription to drinking. The data collection process in the seven WHO-ThaiHealth countries was guided by a study protocol specifically developed to reduce these between-country nuances of meaning as much as possible, for instance by including objective items. Translation and back-translation were employed in each country, as well as pilot testing. This ensured responses had face validity and were meaningful to respondents in each country. However, in the course of archiving, any differences in survey procedure that have come to light are documented in the archive codebooks.

\section{Potential Research Questions and Analyses}

The data collected from the surveys has the potential to be used to answer a number of research questions:

- What is the prevalence of harm attributable to the drinking of others, more specifically, the prevalence of different types of harms in each country? Descriptive statistics will be used to describe the prevalence of harms from known drinkers (i.e., in the household or overall), strangers, co-workers, and harms to the respondent's children. The difference in rates between countries, age groups, and genders will be examined and confidence intervals will be used to check for significant differences between countries.

- What are the relationships between other factors and HTO from drinking? More specific questions include: How is gender/drinking pattern/income associated with HTO from drinking (overall and in specific spheres) across different countries? Predictors of experience of harm will also be calculated using regression models for each country. For instance, logistic regression models predicting the existence of harm from a heavy drinker in the household using demographics, household composition, and own drinking for each country will be generated so that the differences in predictors between countries can be compared. To predict subjective quality of life ratings using the existence of harm, and controlling for demographics, linear regression models will be used again the differences in predictors between countries will be a particular point of interest.

- How do the patterns of relationship between correlates and harm from others' drinking vary between countries? For example, in countries with high per capita consumption figures and high incomes, do the predictors of harm differ from those found in selected low per capita consumption, lowermiddle income countries? A range of cross-national comparisons of patterns of relationship with harm will be possible and enable a wide range of analyses comparing rates of harm per country and within subpopulations. Additionally, regression models can be extended to analyses of the combined datasets, with each country (except one to retain a degree of freedom) specified as a separate dichotomous variable. Combinations of selected similar countries could also be analysed dichotomously in this way. With such methods and the use of interaction terms, analyses can be pursued exploring and taking into account, cross-national patterns and differences in predictors of particular types and characteristics of harm from others' drinking.

- What is the moderating effect of demographics and socio-economic status on the experience of harm attributable to the drinking of others within and between countries? A multi-level analyses of international harm. Pending interest from other countries in contributing data to the database, it is hoped that eventually there will be sufficient power at the country-level to develop nested models predicting harm. In these models, the difference in the relationships between harm and other tested variables 
between countries can be explored and accounted for. While nine countries do not provide sufficient power for nested models, with increasing and ongoing interest and discussion from other countries about contributing data to the study, multi-level analysis should be possible in at minimum specific areas of HTO research within the next 5 years. When there are enough countries to develop these models, it will be possible to investigate numerous research questions that integrate country-level cultural and contextual variables.

\section{Discussion}

While there are a number of subtle variations in measurement between the surveys, the substantive meaning of the vast majority of questions differs only slightly. Thus, this suite of surveys provides the opportunity to make meaningful comparisons across countries with regard to the 10 core areas of the questionnaire: (1) harm from family members' drinking; (2) harm from friends' drinking; (3) harm from co-workers' drinking; (4) harm from strangers' drinking; (5) harm to children from others' drinking; (6) broader questions about harm to others from different drinking patterns of respondents and drinkers; (7) the different ways in which HTO affects men and women; (8) service use and caring patterns of those affected; (9) the effects of HTO on quality of life and wellbeing; and (10) the cost of the harm to others from drinking.

Taking into account alcohol's HTO adds a substantial dimension to the picture of the burden of harm from alcohol, and is potentially an important consideration for national and international policies to improve public health. Recognising the importance of this omission, the WHO decided early in its implementation of the "Global Strategy to Reduce the Harmful Use of Alcohol" to give a high priority to the WHO-ThaiHealth project. In using comparable measures in a diverse range of countries, this project will make an important contribution to general understanding of alcohol problems both at national and international levels. Apart from its utility for policy discussion, the ability to compare rates of reported harms between countries using comparable measures opens up substantial opportunities for comparative and multi-level analyses of cultural differences in the patterning and impact of drinking. This is not only in terms of national differences in circumstances and events, but also in informal and official responses to problematic drinking and its aftermath.

The seven country survey project described in this paper, adding two countries for a total of nine-country dataset, will in itself make additional substantial contributions along these lines. It is already serving as a nucleus attracting further participation and cross-national collaborative work. A dozen high-income countries have collected a shorter "brief assessment" list of questions which are comparable to questions in the present study's surveys, and full studies with substantial comparability to the WHO-ThaiHealth protocols are in progress or planned in several countries. As such, it is hoped that this nine country database will eventually become the core of a much larger project highlighting the range and magnitude of alcohol's HTO on a large international scale.

Investigators planning to conduct a population survey and join in such a project are encouraged to contact this paper's authors.

\section{References}

Alati, R., Al Mamun, A., Williams, G., O'Callaghan, M., Najman, J. M., \& Bor, W. (2006). In utero alcohol exposure and prediction of alcohol disorders in early adulthood: A birth cohort study. Archives of General Psychiatry, 63(9), 1009-1016.

Babor, T., Caetano, R., Casswell, S., Edwards, G., Giesbrecht, N., Graham, K., . . . \& Rossow, I. (2010). Alcohol: No ordinary commodity - Research and public policy (2nd ed.). Oxford: Oxford University Press.

Callinan, S. (2014). Alcohol's harm to others: Quantifying a little or a lot of harm. International Journal of Alcohol and Drug Research, 3(2), 127-133.

Callinan, S., \& Room, R. (2014). Harm, tangible or feared: Young Victorians' adverse experiences from others' drinking or drug use. International Journal of Drug Policy, 25(3), 401-406.

Casswell, S., Harding, J. F., You, R. Q., \& Huckle, T. (2011). Alcohol's harm to others: Self-reports from a representative sample of New Zealanders. The New Zealand Medical Journal, 124(1336), 75-84.

Connor, J., \& Casswell, S. (2009). The burden of road trauma due to other people's drinking. Accident Analysis and Prevention, 41(5), 1099-1103.

Eliany, M., Giesbrecht, N., Nelson, M., Wellman, B., \& Wortley, S. (1992). Alcohol and other drug use by Canadians: A national alcohol and other drugs survey (1989). Ottawa: Health and Welfare Canada.

Graham, K., Bernards, S., Knibbe, R., Kairouz, S., Kuntsche, S., Wilsnack, S. C., . . \& \& Gmel, G. (2011). Alcohol-related negative consequences among drinkers around the world. Addiction, 106(8), 13911405. doi:10.1111/j.1360-0443.2011.03425.x

Graham, K., Bernards, S., Munné, M., Cayetano, C., KerrCorrea, F., Lima, M. C., . . . \& Magri, R. (2008). Comparison of partner physical aggression across ten countries. In K. Graham, S. Bernards, M. Munné, \& S. Wilsnack (Eds.), Unhappy hours: Alcohol and partner aggression in the Americas (pp. 221-248). Washington, D.C, United States: Pan American Health Organisation.

Greenfield, T. K., Ye, Y., Kerr, W., Bond, J., Rehm, J., \& Giesbrecht, N. (2009). Externalities from alcohol consumption in the 2005 US National Alcohol Survey: Implications for policy. International Journal of Environmental Research and Public Health, 6(12), 3205-3224.

Huhtanen, P., \& Tigerstedt, C. (2012). Women and young adults suffer most from other people's drinking. Drug and Alcohol Review, 31(7), 841-846. 
International Wellbeing Group (2013). Personal Wellbeing Index (5th ed.). Melbourne, Australia: Australian Centre on Quality of Life, Deakin University.

Klingemann, H., \& Gmel, G. (Eds.) (2001). Mapping the social consequences of alcohol consumption. Dordrecht, The Netherlands: Kluwer Academic Publishers.

Laslett, A. M., Catalano, P., Chikritzhs, T., Dale, C., Doran, C., Ferris, J., . . . \& Wilkinson, C. (2010). The range and magnitude of alcohol's harm to others. Fitzroy, Victoria: AER Centre for Alcohol Policy Research, Turning Point Alcohol and Drug Centre.

Levine, H. G. (1985). The discovery of addiction: Changing conceptions of habitual drunkenness in America. Journal of Substance Abuse Treatment, 2(1), 43-57.

O'Toole, J., Sinclair, M., \& Leder, K. (2008). Maximising response rates in household telephone surveys. BMC Medical Research Methodology, 8(71). doi: 10.1186/1471-2288-8-71

Rahav, G., Wilsnack, R., Bloomfield, K., Gmel, G., \& Kuntsche, S. (2006). The influence of societal level factors on men's and women's alcohol consumption and alcohol problems. Alcohol and Alcoholism, 41(Suppl 1), i14-i55. doi: 10.1093/alcalc/agl075

Room, R. (2000). Concepts and items in measuring social harm from drinking. Journal of Substance Abuse, 12(1), 93-111. doi: 10.1016/s0899-3289(00)00043-2

Room, R., Ferris, J., Laslett, A. M., Livingston, M., Mugavin, J., \& Wilkinson, C. (2010). The drinker's effect on the social environment: A conceptual framework for studying alcohol's harm to others. International Journal of Environmental Research and Public Health, 7(4), 1855-1871.

Saunders, J. B., Aasland, O. G., Babor, T. F., De la Fuente, J. R., \& Grant, M. (1993). Development of the Alcohol Use Disorders Identification Test (AUDIT): WHO collaborative project on early detection of persons with harmful alcohol consumption-II. Addiction, 88, 791-804.

The EuroQol Group (2009). User Guide: Basic information on how to use EQ-5D (Version 2.0) [User Guide]. Retrieved from http://www.euroqol.org/fileadmin/ user_upload/Documenten/PDF/User_Guide_v2_Marc h_2009.pdf

Wilsnack, R. W., Wilsnack, S. C., Kristjanson, A. F., Vogeltanz-Holm, N. D., \& Gmel, G. (2009). Gender and alcohol consumption: Patterns from the multinational GENACIS project. Addiction, 104(9), 1487-1500. doi: 10.1111/j.1360-0443.2009.02696.x

World Health Organization. (2010). Global strategy to reduce the harmful use of alcohol. Retrieved from http://www.who.int/substance_abuse/alcstratenglishfin al.pdf?ua $=1$ 